\title{
ON MINIMAL THINNESS, REDUCED FUNCTIONS AND GREEN POTENTIALS
}

\author{
by MATTS ESSÉN \\ (Received 3rd January 1991)
}

Let $\Omega$ be an open connected subset of the unit $\operatorname{disc} U$, let $E=U \backslash \Omega$ and let $\left\{\Omega_{k}\right\}$ be a Whitney decomposition of $U$. If $z(Q)$ is the centre of the "square" $Q$, if $T$ is the unit circle and $t=\operatorname{dist} .(Q, T)$, we consider

$$
W(\tau)=W(\tau, E)=\sum\left(\left(1-\left|z\left(Q_{k}\right)\right|\right) /\left|1-\tau \overline{z\left(Q_{k}\right)}\right|\right)^{2}\left(\log \left(4 t_{k} / c\left(E_{k}\right)\right)\right)^{-1}, \tau \in T
$$

where $E_{k}=E \cap Q_{k}$ and $c\left(E_{k}\right)$ is the capacity of $E_{k}$. We prove that the set $E$ is minimally thin at $\tau \in T$ in $U$ if and only if $W(\tau)<\infty$. We study functions of type $W$ and discuss the relation between certain results of Naim on minimal thinness [15], a minimum principle of Beurling [3], related results due to Dahlberg [7] and Sjögren [16] and recent work of Hayman-Lyons [15] (cf. also Bonsall [4]) and Volberg [19]. For simplicity, we discuss our problems in the unit disc $U$ in the plane. However, the same techniques work for analogous problems in higher dimensions and in more complicated regions.

1990 Mathematics subject classification (1985 Revision): 31A15, 31A20, (31B15).

\section{Introduction}

Let $\Omega$ be an open connected subset of the unit disc $U$. We assume that $\Omega$ is regular with respect to the Dirichlet problem. The complement $E=U \backslash \Omega$ is relatively closed in $U$. Let $S H(U)$ denote the class of nonnegative superharmonic functions in $U$. If $h$ is a nonnegative harmonic function in $U$, we consider the reduced function

$$
R_{h}^{E}(z)=\inf \{u(z): u \in S H(U), u \geqq h \text { on } E\}
$$

and the lower regularization

$$
\hat{R}_{h}^{E}(z)=\lim _{w \rightarrow z} \inf R_{h}^{E}(w)
$$

which is superharmonic in $U$ (cf. Helms [12]).

Let us begin by stating a special case of a very general result of Naim (cf. the Corollary p. 234 in [15] and Theorem C in Section 3 below):

Theorem A. $\hat{R}_{1}^{E}$ is a Green potential in $U$ if and only if $E$ is minimally thin at almost all points of the unit circle $T$. 
To define minimal thinness, we introduce the Poisson kernel (or minimal positive harmonic function)

$$
P(\tau, z)=P_{\tau}(z)=(2 \pi)^{-1}\left(1-|z|^{2}\right)|\tau-z|^{-2}, z \in U, \tau \in T .
$$

Definition. The set $E$ is minimally thin at $\tau \in T$ if there exists $z_{0} \in U$ such that $\hat{R}_{h}^{E}\left(z_{0}\right)<h\left(z_{0}\right)$, where $h=P_{\mathrm{r}}$.

This concept was introduced and studied by J. Lelong-Ferrand [14] (for further details, see e.g. [8]). Minimal thinness of a subset $E$ of a half-plane $D$ at a (Martin) boundary point of $D$ is defined in an analogous way.

To state a metric form of Theorem A, we let $\{Q\}$ be a Whitney decomposition of $U$ into approximate "squares" with centres $z(Q)$ such that

$$
\begin{gathered}
Q_{1}^{0} \cap Q_{2}^{0}=\phi, Q_{1} \neq Q_{2} \\
\text { diam. } Q \leqq 3^{-1} \text { dist. }\{z(Q), T\}=t / 3 .
\end{gathered}
$$

(cf. Stein [17]). If $c(E)$ is the capacity of $E$ (cf. Ahlfors [1]), we consider

$$
W(\tau)=W(\tau, E)=\sum\left(\left(1-\left|z\left(Q_{k}\right)\right|\right) /\left|1-\tau \overline{z\left(Q_{k}\right)}\right|\right)^{2}\left(\log \left(4 t_{k} / c\left(E_{k}\right)\right)\right)^{-1},
$$

where $E_{k}=E \cap Q_{k}$. We shall prove that $E$ is minimally thin at $\tau \in T$ if and only if $W(\tau)<\infty$ (cf. Section 1). Applying Theorem A, we obtain:

Theorem 1. $\hat{R}_{1}^{E}$ is a Green potential if and only if $W(\tau)<\infty$ for almost all $\tau \in T$.

We shall also consider.

$$
W_{0}=W_{0}(\tau, E)=\sum_{E \cap Q_{k} \neq \phi}\left(\left(1-\left|z\left(Q_{k}\right)\right|\right) /\left|1-\tau \overline{z\left(Q_{k}\right)}\right|\right)^{2} .
$$

The functions $W$ or $W_{0}$ appear also in two other problems. In the problem considered by Hayman and Lyons [11] (also cf. Bonsall and Walsh [5] and Bonsall [4]), the crucial condition is that we must have $W_{0}(\tau)=\infty$ everywhere on $T$ (more details are given in Section 4). In Section 5, we discuss "boundary layers", a concept introduced by Volberg [19]. A necessary (but not a sufficient) condition for $\Omega=U \backslash E$ to be a boundary layer is that we have $W(\tau)<\infty$ everywhere on $T$.

In the present paper, we wish to study the function $W$ and to show that not only the work of Hayman and Lyons but also other results such as a minimum principle on positive harmonic functions in the plane which is due to Beurling (cf. [3]; also cf. Theorem B in Section 2 below) and the related result of Dahlberg (cf. [7]) can be deduced using the theory of Naim and certain results from potential theory. In our discussions, we walk a middle way between the powerful but rather abstract approach 
of Naim and the classical approach of Hayman and Lyons. For simplicity, we have restricted ourselves to the unit disc. However, the same techniques work for analogous problems in higher dimensions and in more complicated regions. We note that in any dimension, we can use inversion to go from balls to half-spaces. It is a matter of convenience whether we wish to work in a ball or in a half-space.

In the case of the unit disc, it is possible to give a simple direct proof of the result of Naim that we need: this is done in Section 3. In the transition from the Wiener type conditions of [8] to the discussion of the function $W(\tau)$ in the disc, we need condition (3) in Section 1: this is a result of Beurling-Dahlberg type. An independent proof of (3) using a weak $L_{1}$-estimate of Sjögren [16] is given in the Appendix. For readers who are willing to accept some basic results on minimal thinness and the estimate of Sjögren, the proofs in the present paper are self-contained.

I am grateful to Dr. Phil Rippon and his colleagues for comments on an earlier version of the present paper. In particular, the statement and proof of Theorem $4 \mathrm{c}$ is due to them.

\section{A metric criterion for minimal thinness}

In the present section, we prove that a set $E \subset U$ is minimally thin at $\tau \in T$ if and only if $W(\tau)<\infty$.

We consider the conformal mapping $F(z)=(\tau+z) /(\tau-z)$ which maps $U$ conformally onto $D=\{Z=X+i Y: X>0\}$ with $\tau$ going to infinity. Then $E$ will be minimally thin at $\tau$ in $U$ if and only if $G=F(E)$ is minimally thin at infinity in $D$. The Whitney squares $\left\{Q_{k}\right\}$ near $\tau$ in $U$ are mapped onto a collection of Whitney squares $\left\{\tilde{Q}_{k}\right\}$ near infinity in $D$ : let $\tilde{t}_{k}$ and $R_{k}$ be the distances from the centre of $\widetilde{Q}_{k}$ to $\partial D$ and to the origin, respectively. A necessary and sufficient condition for $G$ to be minimally thin at infinity in $D$ is that there exists a measure $v$ on $\partial D$ such that

$$
\sum\left(\tilde{t}_{k} / R_{k}\right)^{2}\left(\log \left(4 \tilde{t}_{k} / c\left(G_{k}\right)\right)^{-1}<\infty,\right.
$$

where $G_{k}=G \cap \widetilde{Q}_{k}$ and we sum over all Whitney squares which do not intersect the set $M(v)=\{Z \in D: P v(Z)>X\}$. Here $P v$ is the Poisson integral of $v$ in $D$ (cf. Theorem 2 in $[8])$. If we can prove that

$$
\sum\left(\tilde{t}_{k} / R_{k}\right)^{2}<\infty
$$

where we sum over all indices $k$ such that $\tilde{Q}_{k} \cap M(v) \neq \emptyset$, it is clear that (1) will be a necessary and sufficient condition for $G$ to be minimally thin at infinity in $D$; this time, we sum over all Whitney squares. Translating this statement to $\tau$ using the mapping $F^{-1}$, we obtain that $W(\tau)$ will be finite and that the metric criterion will be proved.

We replace (2) by the equivalent condition

$$
\iint_{M(v)}\left(1+|Z|^{2}\right)^{-1} d X d Y<\infty
$$


This is a known result (cf. Lemma $I$ in Beurling [3]; the generalization to higher dimensions is given by Dahlberg in [7]). However, since we wish to show that the main result in [3] and the analogue in the plane of the main result in [7] are consequences of the work of Naim, a weak $L_{1}$-estimate of Sjögren given in (20) below and some basic properties of minimal thinness, we give a proof of (3) using these facts in the Appendix.

If in particular $E$ is a union of nonintersecting hyperbolic discs in $U, \log \left(4 t_{k} / c\left(E_{k}\right)\right)$ will be essentially constant and $E$ will be minimally thin at $\tau \in T$ if and only if $W_{0}(\tau)<\infty$ (cf. Section 5 in [11]).

\section{Some results on minimal thinness}

From basic results on minimal thinness, we deduce a key lemma and a minimum principle due to Beurling which we shall call Theorem B. The result of Hayman and Lyons is a consequence of Lemma 1 and Theorem B.

Let $\omega(\cdot, d \zeta)=\omega(\cdot, d \zeta, \Omega)$ be harmonic measure on $\partial \Omega$ : it is defined since we have assumed that $\Omega$ is regular for the Dirichlet problem.

Lemma 1. Assume that $E$ is not minimally thin at $\tau \in T$. Then

$$
P(\tau, z)=\int_{\Gamma} P(\tau, \zeta) \omega(z, d \zeta)
$$

where $\Gamma=\partial \Omega \cap U$. Conversely, if (4) holds at $\tau \in T, E$ is not minimally thin at $\tau$.

Remark. In Hayman and Lyons, it is proved that (4) holds if and only if $W_{0}(\tau)=\infty$ (cf. (3.14) in [11]): they consider the case when $E$ is a union of nonintersecting hyperbolic discs. Our method of proof gives the slightly more general statement given above. For more details we refer to Section 4.

Proof of Lemma 1. Let $\tau \in T$ be such that $E$ is not minimally thin at $\tau$ in $U$. Using the conformal mapping $F$ defined in Section 1 , we move to $D=\{Z=X+i Y: X>0\}$. Then $G=F(E)$ is not minimally thin at infinity in $D$. If $G(R)=G \cap\{|Z|<R\}$, we consider $V_{R}(Z)=\hat{R}_{X}^{G(R)}(Z) \leqq X$. When $R \rightarrow \infty, V_{R}$ increases to a function $V$ which is superharmonic in $D$ and equal to $X$ on $G$. Since $G$ is not minimally thin at infinity in $D$, we have

$$
X=\hat{R}_{X}^{G} \leqq V \leqq X, \quad Z \in D
$$

and it follows that $V(Z)=X$ in $D$. Since $V_{R}$ is bounded, we have 


$$
V_{R}(Z)=\int_{\partial G} V_{R}(\zeta) \omega(Z, d \zeta, D \backslash G(R))
$$

If $R \rightarrow \infty$, it follows that

$$
X=\int_{\partial G}(\mathfrak{R} \zeta) \omega(Z, d \zeta, D \backslash G)
$$

Going back to $U$, we obtain the first part of Lemma 1 .

Conversely, assume that (4) and thus also $\left(4^{\prime}\right)$ holds. From the argument above, it is clear that the right hand member of $\left(4^{\prime}\right)$ is $\hat{R}_{X}^{G}$. Hence we have $X=\hat{R}_{X}^{G}$ which means that $G$ is not minimally thin at infinity and thus that $E$ is not minimally thin at $\tau$. We have proved Lemma 1.

Let $S=\left\{z_{n}\right\}_{1}^{\infty}$ be a sequence of points in $U$ and let

$$
S(\delta)=\bigcup_{1}^{\infty}\left\{z \in U: \rho\left(z, z_{n}\right) \leqq \delta\right\}
$$

where $\rho$ is the hyperbolic metric in $U$ (cf. Beardon [2, Section 7.2]). Following Beurling [3], we call $S$ an equivalence sequence at $\tau \in T$ if for each positive harmonic function $h$ in $U$, the inequalities

$$
h(z) \geqq P(\tau, z), \quad z \in S,
$$

imply that

$$
h(z) \geqq P(\tau, z), \quad z \in U
$$

Theorem B. $S$ is an equivalence sequence at $\tau \in T$ if and only if $S(\delta)$ is not minimally thin at $\tau$ for some positive $\delta$.

Remark. Theorem B is a modified version of the minimum principle of Beurling [3]. Lemma 1 in Hayman and Lyons [11] says that if $S(\delta)$ is minimally thin at $\tau$, then $S$ is not an equivalence sequence at $\tau$.

Proof. To translate minimal thinness into a metric condition, we first form a new sequence by successively deleting points from our sequence that have hyperbolic distance at most $\delta$ from the previous points. Our new sequence $\left\{z_{k}^{\prime}\right\}$ has the following properties:

$$
\begin{gathered}
\rho\left(z_{k}^{\prime}, z_{j}^{\prime}\right)>\delta, k \neq j, \\
S_{1} \subset S_{2} \subset S_{3},
\end{gathered}
$$


where $S_{1}=S\left(\delta / 2,\left\{z_{k}^{\prime}\right\}\right), S_{2}=S\left(\delta,\left\{z_{k}\right\}\right)$ and $S_{3}=S\left(2 \delta,\left\{z_{k}^{\prime}\right\}\right)$.

We claim that these three sets are either all minimally thin at $\tau$ or all not minimally thin at $\tau$. To see this, let us first assume that $S_{1}$ is. minimally thin at $\tau$. Since all hyperbolic discs forming $S_{1}$ are disjoint, the logarithmic term in $W\left(\tau, S_{1}\right)$ is essentially constant and we conclude that $W_{0}\left(\tau, S_{1}\right)$ is finite and that

$$
\sum\left(\left(1-\left|z_{k}^{\prime}\right|\right) /\left|1-\tau \overline{z_{k}^{\prime}}\right|\right)^{2}<\infty
$$

It follows that $W_{0}\left(\tau, S_{3}\right)$ and thus also $W\left(\tau, S_{3}\right)$ will be finite which proves that $S_{3}$ and $S_{2}$ will be minimally thin at $\tau$. Conversely, if $S_{1}$ is not minimally thin at $\tau$, it is clear that the other two sets will not be minimally thin at $\tau$.

We note that if $S\left(\delta_{0}\right)$ is not minimally thin at $\tau$ for some positive $\delta_{0}$, then $S(\delta)$ is not minimally thin at $\tau$ for all $\delta \in\left(0, \delta_{0}\right)$.

The details in the remaining part of the proof of Theorem B are included since similar arguments will be needed in the discussion of Theorem $\mathrm{D}$ and in the proof of Theorem 2 below.

Let us first assume that $S(\delta)$ is minimally thin at $\tau$ which is equivalent to assuming that (9) holds. Again, we move to the halfplane $D=\{Z=X+i Y: X>0\}$, using the conformal mapping $F$ from Section 1. If we define $Z_{k}=X_{k}+i Y_{k}=F\left(z_{k}^{\prime}\right), k=1,2, \ldots$, it follows from (9) that

$$
\sum_{1}^{\infty} X_{k}^{2} /\left(1+Y_{k}^{2}\right)<\infty
$$

Following Beurling, we define

$$
P(Z)=\sum_{1}^{\infty} X X_{k}^{2} /\left(X^{2}+\left(Y-Y_{k}\right)^{2}\right)
$$

Since $P(1)$ is finite by (10), $P$ is a positive harmonic function in $D$ which is finite everywhere. We note that

$$
P\left(Z_{k}\right)>X_{k}, \quad k=1,2, \ldots,
$$

and

$$
P(X) / X=\sum_{1}^{\infty} X_{k}^{2} /\left(X^{2}+Y_{k}^{2}\right) \rightarrow 0, \quad X \rightarrow \infty
$$


Going back to $U$, we obtain (5) for $h(z)=P(F(z))$ on the sequence $\left\{z_{k}^{\prime}\right\}$ (and hence, by Harnack's theorem, on the sequence $\left\{z_{n}\right\}$ ). However, (6) fails for $h$ and so $S^{\prime}$ is not an equivalence sequence.

Conversely, assume that $S(\delta)$ is not minimally thin at $\tau$ and that (5) holds. From Harnack's theorem, we see that there exists a positive constant $C(\delta)$ such that for all $k$ and for all positive harmonic functions in $U$, we have

$$
\begin{gathered}
h(z) \geqq C(\delta) h\left(z_{k}\right), \quad \rho\left(z, z_{k}\right) \leqq \delta, \\
P\left(\tau, z_{k}\right) \geqq C(\delta) P(\tau, z), \quad \rho\left(z, z_{k}\right) \leqq \delta .
\end{gathered}
$$

It follows from (5) that

$$
h(z) \geqq C(\delta)^{2} P(\tau, z), \quad z \in S(\delta) .
$$

If $\varepsilon$ is a given small positive number, we can choose $\delta$ so small that we obtain $C(\delta)^{2} \geqq 1-\varepsilon$. Our assumption that $S(\delta)$ is not minimally thin at $\tau$ implies that

$$
h(z) \geqq(1-\varepsilon) P(\tau, z), \quad z \in U
$$

Since $\varepsilon>0$ can be chosen as small as we like, we obtain (6). This completes the proof of Theorem B.

Remark. Applying criteria for minimal thinness in a halfspace from Essén [8], we can deduce an analogue of Theorem $B$ in higher dimensions.

\section{A theorem on reduced harmonic functions in $\boldsymbol{U}$}

If $E \subset U$ is a given relatively closed set, we write $M=\{\tau \in T: E$ is minimally thin at $\tau\}$, $N=\{\tau \in T: E$ is not minimally thin at $\tau\}$.

Theorem C. Let $h(z)=\int_{T} P(\tau, z) d \mu(\tau)$ be a positive harmonic function in $U$. Then

$$
\hat{R}_{h}^{E}(z)=\int_{N} P(\tau, z) d \mu(\tau)+\int_{M} v(\tau, z) d \mu(\tau)
$$

where $\left.v(\tau, z)=\hat{R}_{P(\tau,}^{E}\right)(z)$ and the second term is a Green potential in $U$.

Remark. For a very general result of this type, we refer to Naim [14, Section 26]. In the case of the unit disc, we give a simple direct proof.

In particular, we see that $\hat{R}_{h}^{E}$ is a Green potential if and only if $\mu(N)=0$. In the case 
$h=1, \mu$ is Lebesgue measure and we obtain Theorem A. Another consequence is that $\hat{R}_{h}^{E}=h$ if and only if $\mu(M)=0$.

Proof. To obtain a representation formula for $\hat{R}_{h}^{E}$, we consider

$$
E_{n}=E \cap\left\{z \in U:|z| \leqq 1-n^{-1}\right\}, \Omega_{n}=U \backslash E_{n}, \Gamma_{n}=\partial \Omega_{n} \cap U
$$

and deduce that

$$
\hat{R}_{h}^{E_{n}}(z)=\int_{\Gamma_{n}} h(\zeta) \omega\left(z, d \zeta, \Omega_{n}\right) \leqq \hat{R}_{h}^{E}(z), z \in U
$$

Letting $n \rightarrow \infty$, we see that the left-hand member increases to a function which is superharmonic in $U$, dominated by $\hat{R}_{h}^{E}$ and which is $h$ on $E$. Thus the limit is $\hat{R}_{h}^{E}$ and we obtain the formula

$$
\hat{R}_{h}^{E}(z)=\int_{\Gamma} h(\zeta) \omega(z, d \zeta, \Omega)=\int_{T} d \mu(\tau) \int_{\Gamma} P(\tau, \zeta) \omega(z, d \zeta, \Omega), z \in U
$$

It $\tau \in N$, we apply Lemma 1 and replace the inner integral by $P(\tau, z)$. If $\tau \in M$ and if $v(\tau, \cdot)$ is a Green potential, then

$$
L(r, v(\tau, \cdot))=\int_{0}^{2 \pi} v\left(\tau, r e^{i \theta}\right) d \theta /(2 \pi)
$$

is a concave function of $\log r$ which decreases to 0 as $r \rightarrow 1-$. It follows that

$$
L\left(r, \int_{M} v(\tau, \cdot) d \mu(\tau)\right)=\int_{M} L(r, v(\tau, \cdot)) d \mu(\tau) \rightarrow 0 \quad \text { as } \quad r \rightarrow 1-
$$

Consequently, the second term in (11) is a Green potential.

It remains to prove that $v(\tau, \cdot)$ is a Green potential for all $\tau \in M$. We consider the Riesz decomposition (cf. Theorem 6.18 in [12])

$$
v(\tau, z)=G \mu_{0}(z)+H(z), z \in U,
$$

where $G \mu_{0}$ is the Green potential of a measure $\mu_{0}$ and $H$ is the largest harmonic minorant of $v(\tau, \cdot)$ in $U$. Since $v(\tau, z)=\hat{R}_{P(\tau, \cdot)}^{E}(z)$ is dominated by the minimal harmonic function $P(\tau, \cdot)$, we have $H=a P(\tau, \cdot)$, where $a \in[0,1]$. Our assumption that $E$ is minimally thin at $\tau$ implies that $a<1$. The function $v_{1}(\tau, \cdot)=v(\tau, \cdot)-a P(\tau, \cdot)$ is nonnegative, superharmonic in $U$ and $(1-a) P(\tau, z)$ for $z \in \Gamma$. Consequently, we have

$$
v_{1}(\tau, z)(1-a)^{-1} \geqq \hat{R}_{P(\tau, \cdot)}^{E}(z)=v(\tau, z), z \in U
$$

and it follows that 


$$
a G \mu_{0}(z) \geqq a(1-a) P(\tau, z), z \in U
$$

If $a$ were positive, we see that a Green potential would dominate a Poisson kernel in $U$ which is impossible. Hence we have $a=0, v(\tau, \cdot)$ is a Green potential and we have proved Theorem C.

We shall use Theorems $B$ and $C$ to give a simple proof of a special case of a result of Dahlberg. Following Dahlberg, we say that a set $\Sigma \subset U$ determines the point measure at $\tau \in T$ if for every positive harmonic function $h$ in $U$ with boundary measure $\mu$, we have

$$
\mu(\{\tau\})=\inf \{h(z) / P(\tau, z): z \in \Sigma\}
$$

Let $\Sigma(\delta)$ be the set $\{z \in U: \rho(z, \zeta) \leqq \delta$ for some $\zeta \in \Sigma\}$. Dahlberg's result is concerned with Liapunov-Dini domains in $R^{N}$, where $N \geqq 3$ (cf. [7]). For the purpose of the present paper, we give a version in the unit disc in the plane.

Theorem D. If $\Sigma \subset U$ and $\tau \in T$, the following conditions on $\Sigma$ are equivalent.

(i) $u$ determines the point measure at $\tau$.

(ii) For some $\delta>0$, we have

$$
\int_{\Sigma(\delta)}|\tau-z|^{-2} d x d y=\infty
$$

(iii) There exists a separated sequence $S=\left\{z_{n}\right\}$ in $\Sigma$ with $\lim z_{n}=\tau$ such that

$$
\sum\left(\left(1-\left|z_{n}\right|\right) /\left|\tau-z_{n}\right|\right)^{2}=\infty
$$

Proof. Let us first assume that (iii) holds and let $E=S(\delta)$. Exactly as in the proof of Theorem B, we can for each $\varepsilon>0$ find a $\delta$ such that if $(h / P)(z)=h(z) / P(\tau, z)$,

$$
\inf _{s}(h / P)(z) \geqq \inf _{E}(h / P)(z) \geqq(1-\varepsilon) \inf _{S}(h / P)(z) .
$$

Thus it suffices to consider the set $E$. By $\left(9^{\prime}\right)$, it is clear that $E$ is not minimally thin at $\tau$. However, at all other points of $T, E$ will be minimally thin. From (11) in Theorem C, we see that

$$
\hat{R}_{h}^{E}(z)=a P(\tau, z)+V(z)
$$

where $a=\mu(\{\tau\})$ and $V$ is a Green potential in $U$. Consequently, for $z \in E$, we have

$$
h(z) / P(\tau, z)=\hat{R}_{h}^{E}(z) / P(\tau, z)=a+V(z) / P(\tau, z) .
$$


It is known (cf. Lelong-Ferrand [14]), that

$$
V(z) / P(\tau, z) \rightarrow 0, z \rightarrow \tau, z \in E \backslash E_{0},
$$

where the exceptional set $E_{0}$ is minimally thin at $\tau$ in $U$. (The results in [14] are given in a halfplane (or a halfspace in higher dimensions). There is no difficulty in transferring them to $U$ since minimal thinness is invariant under conformal mapping in the plane or inversion in balls in higher dimensions).

Since the set $E$ is not minimally thin at $\tau$, the set $E \backslash E_{0}$ is also not minimally thin at $\tau$ and thus non-empty. It follows that

$$
a \leqq \inf _{U}(h / P)(z) \leqq \inf _{E}(h / P)(z)=a .
$$

It is now clear that $S$ and thus also $\Sigma$ determine the point measure at $\tau$.

Conversely, if $\Sigma$ determines the point measure at $\tau \in T$, we see that

$$
h(z) \geqq a P(\tau, z), z \in \Sigma,
$$

implies that

$$
h(z) \geqq a P(\tau, z), z \in U
$$

We wish to apply Theorem B and consider therefore a Whitney decomposition $\left\{Q_{k}\right\}$ of $U$. For each such "square" $Q$ for which $Q \cap \Sigma$ is nonempty, we choose a point in the intersection and find a sequence $S_{1}=\left\{z_{1 n}\right\}$ which is such that $S_{1}\left(\delta_{0}\right) \supset \Sigma$ for some $\delta_{0}>0$. Hence the set $S_{1}\left(\delta_{0}\right)$ determines the point measure at $\tau$, and it follows from Theorem B that $S_{1}(\delta)$ is not minimally thin at $\tau$ for $\delta \in\left(0, \delta_{0}\right)$. Arguing as in the proof of Theorem B, we find a separated subsequence $S$ of $S_{1}$ which is such that $\left(9^{\prime}\right)$ holds.

It remains to prove that we can choose the sequence $S$ is such a way that it will have the limit $\tau$. For simplicity, we move to a half-plane and assume that $\tau$ is at the origin, and that we work with a collection of dyadic Whitney squares. Let $\left\{p_{n}\right\}$ be a sequence of integers such that $\sum_{1}^{\infty} p_{n}^{-1}$ is convergent. It is easy to see that the contribution in our sum $\left(9^{\prime}\right)$ from squares of side $2^{-n}$ and for which the distance to the origin is larger than $p_{n} 2^{-n}$ is the order of magnitude $1 / p_{n}$. Thus we can delete all points in the sequence $S$ of this type without changing the fact that the sum of $\left(9^{\prime}\right)$ is divergent. If we also choose $p_{n}$ in such a way that $p_{n} 2^{-n} \rightarrow 0$ as $n \rightarrow \infty$, the remaining points in $S$ will converge to 0 and the sum $\left(9^{\prime}\right)$ will be divergent.

It is easy to prove that (ii) and (iii) are equivalent. We omit the details.

\section{Bases for positive continuous functions}

Let $B$ be a subclass of the class $C^{+}(T)$ of positive continuous functions on $T$. We say that $B$ is a basis for $C^{+}(T)$ is given $f \in C^{+}(T)$ and $\varepsilon>0$, there exists a sequence $\left\{f_{i}\right\}_{1}^{N} \subset B$ 
and a sequence $\left\{c_{i}\right\}_{1}^{N}$ of positive numbers such that $\left\|f-\sum_{1}^{N} c_{i} f_{i}\right\| \infty<\varepsilon$. In other words, $B$ is a basis for $C^{+}(T)$ if the positive cone spanned by $B$ is dense in $C^{+}(T)$. A subset $S$ of $U$ will be called basic if the set $B=\{P(\cdot, z): z \in S\}$ is a basis for $C^{+}(T)$. If $z_{m, n}=$ $\left(1-2^{-n}\right) \exp \left(2 \pi i m 2^{-n}\right)$, where $1 \leqq n<\infty$ and $0 \leqq m<2^{n}$, we define

$$
Q_{m, n}=\left\{z: 2^{-(n+1)} \leqq 1-|z| \leqq 2^{-n} ; \arg z_{m, n} \leqq \arg z \leqq \arg z_{m+1, n}\right\}
$$

and $S_{m, n}=S \cap Q_{m, n}$. The following result which answers a question of W. Rudin is due to Hayman and Lyons [11, Theorem 2].

Theorem E. A set $S \subset U$ is basic if and only if

$$
W_{1}(\tau)=\sum_{S_{m, n} \neq \phi}\left(1-\left|z_{m, n}\right|\right)^{2}\left|\tau-z_{m, n}\right|^{-2}=\infty
$$

for all $\tau \in T$.

A sequence $\left\{z_{k}\right\}_{1}^{\infty}$ is separated if

$$
\inf \left|\left(z_{k}-z_{j}\right) /\left(1-z_{k} \bar{z}_{j}\right)\right|>0, \quad j \neq k
$$

Hayman and Lyons prove that in Theorem E, it suffices to consider the case when $S$ is a separated sequence. Defining $S(\delta)$ as in Section 2, we see that if $S$ is a separated sequence and if $\delta$ is small enough, $S(\delta)$ will be a collection of nonintersecting hyperbolic discs. If $S=\left\{z_{n}\right\}_{1}^{\infty}$, we consider

$$
W_{2}(\tau)=\sum_{1}^{\infty}\left(\left(1-\left|z_{k}\right|\right) /\left|1-\tau \overline{z_{k}}\right|\right)^{2}, \quad t \in T
$$

It is easy to see that (12) holds if and only if $W_{2}(\tau)=\infty$ for all $\tau \in T$. Thus $S$ will be basic if and only if $S(\delta)$ is not minimally thin at any point on the unit circle (cf. Section 5 in [11]; also cf. Section 1 in the present paper).

The key steps in Hayman and Lyons' proof of Theorem E are [11, Lemma 1 and equation (3.14)], which correspond to Theorem B and Lemma 1 of the present paper.

To give a further connection between the work of Naim and the work of Hayman and Lyons, we let $A$ be a closed subset of $T$ and consider the class $H(U, A)$ of functions $h=h_{1}-h_{2}$, where $h_{1}$ and $h_{2}$ are positive and harmonic in $U$ and have vanishing boundary values on $T \backslash A$. Hayman and Lyons proved (cf. [11, Theorem 3]; cf. also [5, Theorem 10]):

Theorem F. Let $A=T$. Then $S$ is basic if and only if

$$
\sup _{z \in U} h(z)=\sup _{z \in S} h(z) \text { for all } h \in H(U, T) \text {. }
$$

We prove 
Theorem 2. (a) Assume that $S \subset U$ and that $S(\delta)$ is not minimally thin at any point of A. Then

$$
\sup _{z \in U} h(z)=\sup _{z \in S} h(z), \text { for all } h \in H(U, A)
$$

(b) Let $S_{n}^{\prime}(\delta)$ be the radial projection onto $T$ of the hyperbolic disc $S_{n}(\delta)=\left\{z \in U: \rho\left(z, z_{n}\right) \leqq\right.$ $\delta\}$. Assume that $S_{n}^{\prime}(\delta) \cap A \neq \emptyset, n=1,2, \ldots$ Then (15) is a necessary and sufficient condition for $S(\delta)$ not to be minimally thin at any point of $A$.

We need (cf. Lemma 5 in [11])

Lemma 2. Let $h$ be a positive harmonic function in $U$. Suppose that $\delta \in\left(0, \frac{1}{2}\right)$ is given and that $z_{1}$ and $z_{2}$ are two points in $U$ such that $\rho\left(z_{1}, z_{2}\right)<\delta$. Then there is an absolute constant $C$ such that

$$
\left|h\left(z_{1}\right)-h\left(z_{2}\right)\right|<C \delta h\left(z_{1}\right)
$$

Proof of Theorem 2. (a) Without loss of generality, we can assume that the hyperbolic discs that form $S(\delta)$ are pairwise disjoint (cf. the proof of Lemma B). Defining $g_{n}(z)=\int_{S_{n}(\delta)} \omega(z, d \zeta)$, we note that $\sum g_{n} \leqq 1$.

Let us first use Lemma 2 to conclude that

$$
\begin{aligned}
& h_{1}(\zeta) \leqq h_{1}\left(z_{n}\right)(1+C \delta), \zeta \in S_{n}(\delta), \\
& h_{2}(\zeta) \geqq h_{2}\left(z_{n}\right)(1-C \delta), \zeta \in S_{n}(\delta) .
\end{aligned}
$$

Under our assumptions, it follows from Theorem $C$ with $E=S(\delta)$ that

$$
\begin{aligned}
& h_{1}(z)=\hat{R}_{h_{1}}^{E}(z)=\sum_{1}^{\infty} \int_{S_{n}(\delta)} h_{1}(\zeta) \omega(z, d \zeta) \leqq(1+C \delta) \sum_{1}^{\infty} g_{n}(z) h_{1}\left(z_{n}\right), \\
& h_{2}(z)=\hat{R}_{h_{2}}^{E}(z)=\sum_{1} \int_{S_{n}(\delta)} h_{2}(\zeta) \omega(z, d \zeta) \geqq(1-C \delta) \sum_{1}^{\infty} g_{n}(z) h_{2}\left(z_{n}\right),
\end{aligned}
$$

and that

$$
h_{1}(z)(1+C \delta)^{-1}-h_{2}(z)(1-C \delta)^{-1} \leqq \sum_{1}^{\infty} g_{n}(z) h\left(z_{n}\right) \leqq \sup _{n} h\left(z_{n}\right)
$$

Letting $\delta \rightarrow 0$, we obtain (15).

(b) It suffices to prove that if $S(\delta)$ is minimally thin at $\tau_{0} \in A$, then (15) does not hold. In 
each disc $S_{n}(\delta)$, we choose $z_{n}^{*}$ such that $z_{n}^{*} /\left|z_{n}^{*}\right| \in A$. If $S_{1}=\left\{z_{n}^{*}\right\}_{1}^{\infty}$, it is clear that $S_{1}(\delta)$ is minimally thin at $\tau_{0}$. We use the construction in the proof of Theorem $B$ to build $\mathbf{a}$ positive harmonic function satisfying the conditions of Theorem B with $S$ replaced by $S_{1}$. Furthermore, the support of the associated measure of $h$ on $T$ is contained in the radial projection of $S_{1}$ onto $T$. It follows that $h \in H(U, A)$. We can now use an argument from Section 5 in [11]. We have

$$
\begin{gathered}
h(z) \geqq P\left(\tau_{0}, z\right), z \in S_{1}, \\
h\left(r \tau_{0}\right) / P\left(\tau_{0}, r \tau_{0}\right) \rightarrow 0, r \rightarrow 1-.
\end{gathered}
$$

Hence the function $h_{1}(z)=P\left(\tau_{0}, z\right)-h(z)$ belongs to $H(U, A)$ and is nonpositive on $S_{1}$ but positive at points in $U$ near $\tau_{0}$. This contradicts $(15)$ and completes the proof of Theorem 2.

\section{Boundary layers}

The following concept has been introduced by Volberg [19]. Let $E$ be as in the introduction and assume furthermore that $\Omega=U \backslash E$ is connected. We say that $\Omega$ is a boundary layer if there exists a constant $c>0$ such that $\omega(0, I) \geqq c|I|$ for all arcs $I \subset T$, where $\omega(0, I)$ is the harmonic measure of the arc $I$ in the domain $\Omega$ evaluated at the origin. (Instead of the origin, we can of course use any other fixed point in $\Omega$.)

The purpose of the present section is to show how certain results of Volberg follow from our arguments and thus to illustrate the relationship between Theorem $A$ and the work of Volberg.

If $\Gamma=\hat{\partial} \Omega \cap U$, we have

$$
h(0)=\left(\int_{\Gamma}+\int_{T}\right) h(\zeta) \omega(0, d \zeta)
$$

for all harmonic functions $h$ which are also continuous in $\bar{U}$. If $\Omega$ is a boundary layer, we have

$$
\int_{T} h(\zeta) \omega(0, d \zeta) \geqq c \int_{T} h(\zeta) d \theta /(2 \pi)=c h(0)
$$

so that

$$
\int_{\Gamma} h(\zeta) \omega(0, d \zeta) \leqq(1-c) h(0)
$$

for all such functions $h$. Choosing $h(z)=P(\tau, r z)$, where $r \in(0,1)$, we have

$$
\int_{\Gamma} P(\tau, r \zeta) \omega(0, d \zeta) \leqq 1-c
$$


Letting $r \rightarrow 1-$, we deduce using Fatou's theorem that

$$
P^{\omega}(\tau):=\int_{\Gamma} P(\tau, \zeta) \omega(0, d \zeta) \leqq 1-c,
$$

for all $\tau \in T$. It is clear that this is also a sufficient condition for $\Omega$ to be a boundary layer.

Volberg proves the following results.

(i) Let $\frac{1}{2} U \subset \Omega$. Then there exists a constant $A_{1}$ such that

$$
P^{\omega}(\tau) \leqq A_{1} W(\tau), \tau \in T
$$

Thus, if $\sup W(\tau) \leqq(1-c) / A_{1},(17)$ holds for all $\tau \in T$ and $\Omega$ is a boundary layer.

(ii) There exist positive absolute constants $A_{2}$ and $q_{0}$ such that if (17) holds for all $\tau \in T$ with $q=1-c \leqq q_{0}$, then $\sup W(\tau) \leqq A_{2} q$. If $S$ is a Stolz domain and $E \subset S$, then $W(\tau) \leqq A_{2} P^{\omega}(\tau), \tau \in T$, and the following assertions are equivalent:

(a) $\Omega$ is a boundary layer.

(b) $\sup _{\tau} W(\tau)<\infty$.

(iii) If the series defining $W$ converges uniformly on $T$, then $\Omega$ has certain nice properties which define what Volberg calls a good boundary layer (for details, we refer to [19]). Assuming that $\frac{1}{2} U \subset \Omega$, we prove

Theorem 3. (a) A necessary but not a sufficient condition for $\Omega$ to be a boundary layer is that $E$ is minimally thin everywhere on $T$.

(b) Assume that the series defining $W$ converges uniformly on $T$. Then $E$ is minimally thin everywhere on $T$ and $\Omega$ is a boundary layer.

Proof. (a) If $E$ is not minimally thin at $\tau \in T$, it follows from Lemma 1 that $P^{\omega}(\tau)=1$. Hence (17) doesn't hold and $\Omega$ is not a boundary layer.

To give an example showing that minimal thinness everywhere on $T$ is not a sufficient condition for $\Omega$ to be a boundary layer, we consider

$$
E=U_{1}^{\infty} E_{n}, \text { where } E_{n}=\left\{z=e^{i \theta}\left(1-2^{-2 n}\right): 2^{-n-1} \leqq \theta \leqq 2^{-n}\right\} .
$$

This set is minimally thin everywhere on $T$ except possibly at 1 .

We have $W(1) \leqq$ Const. $\sum_{1}^{\infty} 2^{-n}<\infty$. This is clear since each set $E_{n}$ is contained in the union of $2^{n}$ closed Whitney squares $Q_{k}^{(n)}$ and $4 t_{k}^{(n)} / c\left(E_{n} \cap Q_{k}^{(n)}\right)$ is essentially constant for all these squares. From the criterion in Section 1, we see that $E$ is minimally thin also at 1 .

We wish to prove that there is a sequence of arcs on $T$ tending to 1 which have small 
harmonic measures at the origin in $\Omega=U \backslash E$. The first step in the proof is the following lemma which for simplicity is stated in the right half-plane $D$.

Lemma 3. Let $a$ and $b$ be positive numbers which are such that $b-a \gg 1$. We consider the domain

$$
D(b)=D \backslash\{z=x+i y: x=1,|y| \leqq b\},
$$

the box $B_{t}=\{z=x+i y: 0<x \leqq 1,|y| \leqq t\}$ and the interval $J=\{z=i y:|y|<a\}$. Then there is an absolute constant $C$ such that

$$
\omega(z, J, D(b)) \leqq C \exp (-\pi(b-a)), z \in D(b) \backslash B_{b},
$$

where the left-hand side denotes the harmonic measure of $J$ in the domain $D(b)$.

Proof. Let $h$ be harmonic in $D(b) \backslash B_{a}$ with boundary values 1 on $\partial B_{a} \cap\{z=$ $x+i y: 0<x<1\}$ and 0 on the rest of the boundary. It is clear that $h$ majorizes $\omega(z, J, D(b))$ in $D(b) \backslash B_{a}$. The estimate in (18) is a simple consequence of standard estimates of harmonic measure (cf. the Corollary p. 116 in Tsuj [18]).

We return to the set $E$ defined above. Let $J_{n}$ be the radial projection of the middle half of the arc $E_{n}$ onto $T$. It follows from Lemma 3 that

$$
\omega\left(0, J_{n}, \Omega\right) \leqq C \exp \left(-\pi 2^{n-3}\right),
$$

and that

$$
\omega\left(0, J_{n}, \Omega\right) /\left|J_{n}\right| \leqq C 2^{n} \exp \left(-\pi 2^{n-3}\right) \rightarrow 0, n \rightarrow \infty
$$

We have proved that $\Omega=U \backslash E$ is not a boundary layer and that the set $E$ is minimally thin at all points of $T$.

(b) Assume that $\frac{1}{2} U \subset \Omega$. If the series defining $W$ is uniformly convergent on $T, E$ will be minimally thin everywhere on $T$ (cf. Section 1) and it follows from Lemma 1 and the maximum principle that

$$
P^{\omega}(\tau)=\int_{\Gamma} P(\tau, \zeta) \omega(0, d \zeta)<P(\tau, 0)=1, \tau \in T
$$

If (17) doesn't hold, there exists a sequence $\left\{\tau_{n}\right\}$ such that $P^{\omega}\left(\tau_{n}\right) \rightarrow 1$ as $n \rightarrow \infty$. Without loss of generality, we can assume that $\tau_{n}=\exp \left(i \theta_{n}\right) \rightarrow 1$, where $\left\{\theta_{n}\right\}$ is a sequence decreasing to 0 . Let $\varepsilon>0$ be given. We know that $P^{\omega}(1)<1$. Since the series defining $W$ converges uniformly, we can find a neighbourhood $O$ of 1 in $U$ such that

$$
W(\tau, O)<\varepsilon \text { for all } \tau \in \bar{O} \cap T
$$


where $W(\tau, O)$ is defined as the sum over all Whitney squares intersecting $O$ in the sum defining $W$. We write

$$
W(\tau)=W(\tau, O)+(W(\tau)-W(\tau, O))=\left(W_{1}+W_{2}\right)(\tau)
$$

If $P_{1}^{\omega}(\tau)=\int_{\Gamma \cap o} P(\tau, \zeta) \omega(0, d \zeta, \Omega)$, we define $P_{2}^{\omega}=P^{\omega}-P_{1}^{\omega}$ and $E_{1}=E \cap \bar{O}$. It is known that

$$
\omega(\cdot, d \zeta, U \backslash E) \leqq \omega\left(\cdot, d \zeta, U \backslash E_{1}\right), \zeta \in \Gamma \cap \bar{O} .
$$

If $\omega=\omega(\cdot, d \zeta, U \backslash E)$ and $\omega^{\prime}=\omega\left(\cdot, d \zeta, U \backslash E_{1}\right)$, we have

$$
P_{1}^{\omega}(\tau) \leqq P^{\omega^{\prime}}(\tau), \tau \in T
$$

and we can use Volberg's estimate (i) to deduce that

$$
\lim \sup _{n \rightarrow \infty} P_{1}^{\omega}\left(\tau_{n}\right) \leqq A_{1} \lim \sup _{n \rightarrow \infty} W_{1}\left(\tau_{n}\right) \leqq A_{1} \varepsilon,
$$

so that

$$
\lim _{n \rightarrow \infty} P_{2}^{\omega}\left(\tau_{n}\right)=P_{2}^{\omega}(1) \leqq P^{\omega}(1)
$$

If $\varepsilon$ is small enough, we conclude that $1=\lim _{n \rightarrow \infty} P^{\omega}\left(\tau_{n}\right)<1$ which is impossible. From the contradiction, we see that there is no such sequence $\left\{\tau_{n}\right\}$. Hence (17) holds and $\Omega$ is a boundary layer. This concludes the proof of Theorem 3 .

Corollary. Assume that

$$
\sum\left(\log \left(4 t_{k} / c\left(E_{k}\right)\right)^{-1}<\infty\right.
$$

Then $E$ is minimally thin everywhere on $T$ and $\Omega$ is a boundary layer.

Proof. If (18) holds, it is easy to see that the series defining $W$ is uniformly convergent. It follows from Theorem 3 that $\Omega$ is a boundary layer.

As mentioned in the introduction, the results on minimal thinness hold also in $R^{d}$, $d \geqq 3$. It should be possible to prove analogues of the Hayman-Lyons and the Volberg results in higher dimensions.

\section{Concluding remarks}

Let $S=\left\{z_{n}\right\}$ be a separated sequence in $U$ and let $E=S(\delta)$ be defined as in Section 2, so that $S(\delta)$ in the union of nonintersecting hyperbolic discs. Let us consider the following statements. 
(A) $\Omega=U \backslash E$ is a boundary layer.

(B) $\hat{R}_{1}^{E}$ is a Green potential.

(C) $\sup _{\mathrm{r} \in T} W_{0}(\tau) \leqq M<\infty$.

(D) $S$ is an interpolating sequence (cf. Ch. VII in [10]).

(E) $W_{0}(\tau)<\infty$ a.e. on $T$.

\section{Theorem 4.}

(a) $(A) \Rightarrow(B)$

(b) $(C) \Rightarrow(D)$.

(c) $(D) \Rightarrow(E)$.

The implications can not be reversed.

Proof. (a) This conclusion is immediate from Theorems 3 and A.

Remark. If $q=1-c$ is small enough, we have $(A) \Rightarrow(C)$ (cf. Volberg's result (ii)). We also note that according to Theorem 1 , we have $(B) \Leftrightarrow(E)$.

(b) For simplicity, we give the argument in the halfplane $D=\{\operatorname{Rez}>0\}$. Thus, if $\left\{z_{j}\right\}$ is a separated sequence in $D$ and

$$
\sup _{t \in R} \sum\left(x_{j} /\left|i t-z_{j}\right|\right)^{2} \leqq M
$$

we wish to prove that $\left\{z_{j}\right\}$ is an interpolating sequence. According to Carleson's theorem, it suffices to prove that $\lambda=\sum x_{j} \delta_{z_{j}}$ is a Carleson measure (cf. Theorem VII.1.1. in [10]). We choose a square $Q=[0, L] \times[a, a+L]$ and note that

$$
\int_{a}^{a+L} \sum_{z_{j} \in Q}\left(x_{j} /\left|i t-z_{j}\right|\right)^{2} d t \geqq \text { Const. } \sum_{z_{j} \in Q} x_{j} .
$$

Using (19), we conclude that $\sum_{z_{j} \in Q} x_{j} \leqq$ Const.L. We have proved that $(C) \Rightarrow(D)$.

An example showing that the implication can not be reversed is given by $z_{j}=1-2^{-j}$, $j=1,2, \ldots$ This is clearly an interpolating sequence. On the other hand, $W_{0}(1)=\infty$ and (C) does not hold.

(c) If $S$ is an interpolating sequence, we consider the Blaschke product

$$
B(z)=B(z, S)=\prod_{1}^{\infty}\left(-\left|z_{n}\right| / z_{n}\right)\left(z-z_{n}\right) /\left(1-z \overline{z_{n}}\right) .
$$

If $\rho\left(z, z_{p}\right)<\delta$, there exists an absolute constant $C$ such that

$$
\left|z-z_{p}\right|<C \delta\left(1-\left|z_{p}\right|\right)
$$


and we have

$$
|B(z)| \leqq C \delta, \delta\left(z, z_{p}\right)<\delta .
$$

If $E=S(\delta)$ and $u=\hat{R}_{1}^{E}$, we see that if $\delta$ is small enough,

$$
u(z) \leqq\left(\log (C \delta)^{-1}\right)^{-1} \log (1 /|B(z)|), z \in U .
$$

Thus $u$ is dominated by a Green potential and it follows that $u$ itself must be a Green potential. Applying Theorem 1, we obtain $(E)$.

To see that the converse is false, we consider the sequence $S$ defined by

$$
z_{k, n}=\left(1-2^{-n} \exp \left(2 \pi i k 2^{-n}\right)\right), \leqq k \leqq 2^{n}, \quad n=1,2, \ldots
$$

which is not interpolating, since we have

$$
\sum_{k, n}\left(1-\left|z_{k, n}\right|\right)=\sum_{n=1}^{\infty} 1=\infty
$$

If $\delta$ is small, $E=S(\delta)$ is a union of nonintersection hyperbolic discs and $E$ is minimally thin everywhere on $T$ except possibly at $z=1$. However, a computation shows that $W_{2}(1)$ is finite, and so $E$ is minimally thin everywhere on $T$ but $S$ is not an interpolating sequence.

Let us finally discuss the relationship between a sequence $S=\left\{z_{n}\right\}$ and the function

$$
V_{2}(\tau)=\sum_{1}^{\infty}\left(\left(1-\left|z_{n}\right|\right) /\left|1-\tau \overline{z_{n}}\right|\right), \tau \in T
$$

which should be compared to $W_{2}(\tau)$. A classical theorem of Frostman [9] says that the Blaschke product $B(z, S)$ and all of its subproducts have angular limits of modulus 1 at $\tau$ if and only if $V_{2}(\tau)$ is finite. J. S. Hwang has proved that $V_{2}(\tau)$ is finite if and only if the Green potential $-\log |B(z, S)|$ has the rarefied fine limit 0 at $\tau$ (cf. [13]). Minimal thinness and rarefiedness are two different ways of describing "smallness" of a set near a boundary point (cf. [8]).

We hope to continue the study of these problems in a later paper, both in the plane and in higher dimensions.

\section{Appendix}

We return to questions discussed in Section 1. We work in the half-plane $D=\{z=$ $x+i y: x>0\}$ and consider the Poisson integral $P v$ defined by

$$
P v(z)=\pi^{-1} \int z|z-i t|^{-2} d v(t)
$$


and the set $M(v)=\{z \in D: P v(z) \geqq x\}$.

To prove that (3) holds, we first localize the problem and consider

$$
\begin{aligned}
& I_{n}=\left\{t \in \partial D: 2^{n} \leqq|t|<2^{n+1}\right\}, \\
& D_{n}=\left\{z \in D: 2^{n} \leqq|z|<2^{n+1}\right\}
\end{aligned}
$$

the restriction $d v_{n}$ of $d v$ to $I_{n}$ and $P_{n}(z)=P v_{n}(z)$. If $n_{0}$ is a fixed positive integer and $|z|$ is large, the sum $\sum_{-\infty}^{n_{0}} P_{n}(z)$ will be an error term in our estimates which will be small, and hence the convergence of the integral in (3) depends only on the behaviour of $v$ near infinity. Thus we can assume that the support of $d v$ is contained in $\partial D \cap\left\{|z| \geqq 2^{n_{0}}\right\}$ and that

$$
\int\left(1+t^{2}\right)^{-1} d v(t) \leqq \pi / 32
$$

If $z \in D_{n}$, we have

$$
\begin{aligned}
& \sum_{k \leqq n-2} P_{k}(z) \leqq \sum_{k \leqq n-2} 4 \pi^{-1}|z|^{-2}|| v_{k}|| x \leqq x / 4, \\
& \sum_{k \geqq n+2} P_{k}(z) \leqq \sum_{k \geqq n+2} 4 \pi^{-1} x \int_{I_{k}} t^{-2} d v(t) \leqq x / 4
\end{aligned}
$$

and it follows that

$$
\left\{z \in D_{n}: P v(z) \geqq x,\right\} \subset\left\{z \in D_{n}:\left(P_{n-1}+P_{n}+P_{n+1}\right)(z) \geqq x / 2\right\}
$$

With $z^{\prime}=z / 2^{n}$ and $d \lambda_{n}(t)=2^{-2 n}\left(d v_{n-1}+d v_{n}+d v_{n+1}\right)\left(2^{n} t\right)$, this latter set is a scaling by $2^{n}$ of

$$
\left\{z^{\prime} \in D_{n} / 2^{n}: P \lambda_{n}\left(z^{\prime}\right) \geqq x^{\prime} / 2\right\}
$$

To complete the proof, we note that there is an absolute constant $C$ such that if

$$
M(\lambda)=\{z \in D: P \lambda(z) \geqq x\}
$$

then

$$
|M(\lambda)|=\iint_{M(\lambda)} d x d y \leqq C\|\lambda\|
$$

(cf. Theorem 2 in Sjögren [16]). It follows from the discussion above that we have

$$
\iint_{M(v)}\left(1+|z|^{2}\right)^{-1} d x d y \leqq C \sum\left\|\lambda_{n}\right\| \leqq C_{1} \int\left(1+t^{2}\right)^{-1} d v(t)<\infty .
$$


This finishes the proof of (3).

\section{REFERENCES}

1. L. V. AhLfors, Conformal invariants, Topics in Geometric Function Theory (McGraw-Hill, New York 1973).

2. A. F. Beardon, The geometry of discrete groups (Springer-Verlag, New York, 1983).

3. A. Beurling, A minimum principle for positive harmonic functions, Ann. Acad. Sci. Fenn. Ser. A I Math. 372 (1965).

4. F. F. Bonsall, Domination of the supremum of a bounded harmonic function by its supremum over a countable subset, Proc. Edinburgh Math. Soc. 30 (1987), 471-477.

5. F. F. Bonsall and D. Walsh, Vanishing $l^{1}$-sums of the Poisson kernel, and sums with positive coefficients, Proc. Edinburgh Math. Soc. 32 (1989), 431-447.

6. E. F. Collingwood and A. J. Lohwater, The theory of cluster sets (Cambridge University Press, 1966).

7. B. Dahlberg, A minimum principle for positive harmonic functions, Proc. London Math. Soc. 33 (1976), 238-250.

8. M. Essén, On Wiener conditions for minimally thin and rarefied sets, in Complex Analysis (ed. J. Hersch and A. Huber, Birkhäuser Verlag, Basel 1988), 41-50.

9. O. Frostman, Sur les produits de Blaschke, K. Fysiogr. Sällsk, Lund Förh. 12 (1939), 1-14.

10. J. B. Garnett, Bounded Analytic Functions (Academic Press, New York, 1981).

11. W. K. Hayman and T. J. Lyons, Bases for positive continuous functions, J. London Math. Soc. (2) 42 (1990), 292-308.

12. L. L. Helms, Introduction to potential theory (Wiley-Interscience, New York-London 1969).

13. J. S. Hwang, On boundary behaviour of Blaschke products, Analysis 6 (1986), 317-338.

14. J. Lelong-Ferrand, Étude au voisinage de la frontière des fonctions surharmoniques positives dans un demi-espace, Ann. Sci. École Norm. Sup. (3) 66 (1949), 125-159.

15. L. Naim, Sur le rôle de la frontière de R. S. Martin dans la théorie du potentiel, Ann. Inst. Fourier 7 (1957), 183-281.

16. P. SJögren, Weak $L_{1}$ characterizations of Poisson integrals, Green potentials and $H^{p}$ spaces, Trans. Amer. Math. Soc. 233 (1977), 179-196.

17. E. M. Stein, Singular integrals and differentiability properties of functions (Princeton University Press, 1970).

18. M. Tsus, Potential theory in modern function theory (Maruzen, Tokyo, 1959).

19. A. L. Volberg, A criterion on a subdomain of the disc to have its harmonic measure comparable with Lebesgue measure (USSR Academy of Sciences. Steklov Mathematical Institute, Leningrad department, LOMI preprints E-2-89). Also Proc. Amer. Math. Soc., to appear.

UPPSALA UNIVERSITY

Department of Mathematics

Box 480

S-75106 UPPSALA

SWEDEN 